\title{
Comunicação neuroimunológica no desenvolvimento de doenças mentais
}

\author{
Neuroimmunological communication in the development of \\ mental diseases
}

\author{
Comunicación neuroinmunológica en el desarrollo de \\ enfermedades mentales
}

\author{
Laura Cabral Peixoto ${ }^{1}$, Vanessa de Albuquerque Brito ${ }^{1}$, Rhaldney Kaio \\ Silva Galvão², Michelle Melgarejo da Rosa ${ }^{3}$ \\ 1.Graduanda de Farmácia do Centro Universitário UniFBV Wyden, Recife-PE, Brasil. \\ 2.Graduando de Biomedicina do Centro Universitário UniFBV Wyden, Recife-PE, Brasil. \\ 3.Doutora em Neurobiologia, Professora Adjunta da Universidade Federal de Pernambuco \\ (UFPE), Departamento de Bioquímica, Recife-PE, Brasil.
}

\begin{abstract}
Resumo
Introdução. Atualmente, sabe-se que os mecanismos cerebrais como o desenvolvimento de memórias traumáticas, sofrem interferência do sistema imune e, portanto, são modulados por processos imunológicos. Nesta linha, estudos relatam o aumento ou a diminuição de mediadores inflamatórios em pessoas com déficits cognitivos ou distúrbios de humor como depressão. Objetivo. Explanar a influência do sistema imunológico na patofisiologia do Sistema Nervoso Central (SNC). Método. Revisão bibliográfica a partir de buscas em bancos de dados acadêmicos e dados epidemiológicos como SCIELO, SCIENCE DIRECT, PUBMED e DATASUS. A consulta foi realizada utilizando como palavras-chave: "doenças neurodegenerativas", "doenças psicológicas", "sistema imunológico" "neuroimunomodulação" e "mecanismos cerebrais". Artigos na língua inglesa e portuguesa, publicados nos últimos 15 anos foram considerados. Resultados. Inúmeros estímulos provenientes do SNC são capazes de modular uma resposta imune e vice-versa. O eixo Hipotálamo-Pituitária Adrenal (HPA) é um dos responsáveis nessa troca de informação. Também participam desta relação alguns hormônios como: endorfinas, prostaglandinas, tireotrofinas, hormônio do crescimento e o Sistema Nervoso Autônomo Simpático (SNAS). Além disso, a ativação do eixo HPA e a consequente produção dos hormônios glicocorticoides durante o processo de estresse são um dos principais mecanismos responsáveis pelas alterações da resposta imune corporal. Conclusão. Insere-se uma importante comunicação entre o SNC e o sistema imune, mostrando que a neuroimunomodulação, entra hoje, com uma nova oportunidade terapêutica para o esquecimento de fatos traumáticos, distúrbios de humor e déficits cognitivos.
\end{abstract}

Unitermos. Doenças neurodegenerativas; neuroimunomodulação; doenças mentais

\begin{abstract}
Introduction. Currently, it is known that brain mechanisms, such as the development of traumatic memories, suffer interference from the immune system and, therefore, are modulated by immunological processes. In this view, studies report an increase or decrease in inflammatory mediators in people with cognitive deficits or mood disorders such as depression, for instance. Objective. To explain the influence of the immune system on the pathophysiology of the Central Nervous System (CNS). Method. Literature review based on searches in academic databases and epidemiological data such as SCIELO, SCIENCE DIRECT, PUBMED, and DATASUS. The search was carried out using the keywords: "neurodegenerative diseases", "psychological diseases", "immune system" "neuroimmunomodulation" and "brain mechanisms". Articles in English and Portuguese, published in the last 15 years, were considered. Results. Countless stimuli from the CNS are capable of modulating an immune response and vice versa. The Hypothalamus-Pituitary Adrenal (HPA) axis is one of those responsible for this exchange of information. Also involved in this relationship are some
\end{abstract}


hormones such as: endorphins, prostaglandins, thyrotrophins, growth hormone and the Sympathetic Autonomic Nervous System (SNAS). In addition, the activation of the HPA axis and the consequent production of glucocorticoid hormones are the hallmark mechanisms for changes in the body's immune response. Conclusion. An important communication between the CNS and the immune system is inserted, showing that neuroimmunomodulation enters today, with a new therapeutic opportunity for forgetting traumatic facts, mood disorders and cognitive deficits.

Keywords. Neurodegenerative diseases; neuroimmunomodulation; mental disease

\section{Resumen}

Introducció. Actualmente se sabe que los mecanismos cerebrales, como el desarrollo de recuerdos traumáticos, sufren interferencias del sistema inmunológico y, por tanto, son modulados por procesos inmunológicos. En esta línea, los estudios reportan un aumento o disminución de mediadores inflamatorios en personas con déficits cognitivos o trastornos del estado de ánimo como la depresión. Objetivo. Explicar la influencia del sistema inmunológico en la fisiología del Sistema Nervioso Central (SNC). Método. Revisión de la literatura basada en búsquedas en bases de datos académicas y datos epidemiológicos como SCIELO, SCIENCE DIRECT, PUBMED y DATASUS. La consulta se realizó utilizando como palabras clave: "enfermedades neurodegenerativas", "enfermedades psicológicas", "sistema inmunológico", "neuroinmunomodulación" y "mecanismos cerebrales". Se consideraron artículos en inglés y portugués publicados en los últimos 15 años. Resultados. Innumerables estímulos del SNC son capaces de modular una respuesta inmune y viceversa. El eje Hipotálamo-Hipofisario Adrenal (HPA) es uno de los responsables de este intercambio de información. También participan en esta relación algunas hormonas como: endorfinas, prostaglandinas, tirotrofinas, hormona del crecimiento y el Sistema Nervioso Autonómico Simpático (SNAS). Además, la activación del eje HPA y la consecuente producción de hormonas glucocorticoides durante el proceso de estrés son uno de los principales mecanismos responsables de los cambios en la respuesta inmunitaria del organismo. Conclusión. Se inserta una importante comunicación entre el SNC y el sistema inmunológico, mostrando que la neuroinmunomodulación entra hoy, con una nueva oportunidad terapéutica para el olvido de hechos traumáticos, trastornos del estado de ánimo y déficits cognitivos.

Palabras clave. Enfermedades neurodegenerativas; neuroinmunomodulación; enfermidades mentales

Trabalho realizado no Centro Universitário UniFBV/Wyden, Recife-PE, Brasil.

\section{INTRODUÇÃO}

Há algum tempo, acreditava-se que o Sistema Nervoso Central (SNC) possuía a característica de ser imunoprivilegiado, sendo regulado de maneira independente a outros sistemas, como o sistema imune, por exemplo. No entanto, atualmente sabe-se que o SNC é modulado de maneira coordenada pelo sistema imune e que a 
neuroimunoregulação é a barreira existente entre saúde e doença ${ }^{1}$.

Há um aumento de mediadores inflamatórios, como citocinas inflamatórias, em pacientes com distúrbios de humor e, sabe-se que a produção cerebral excessiva de IL-1 e IL-6 in vivo, é capaz de provocar déficits cognitivos e mnemônicos ${ }^{2}$. Cresce evidências de que a fisiopatologia de doenças neurodegenerativas como Alzheimer e Parkinson é proporcional à dimensão do processo inflamatório local. Verifica-se um acúmulo de células astrocitárias ao redor dos emaranhados neurofibrilares característicos da patogenia da doença de Alzheimer ${ }^{3}$. Diversos estudos demonstram a importante influência do sistema imunológico na fisiologia do sistema nervoso central, mostrando que doenças psicológicas e neurodegenerativas são gerenciadas por células imunológicas ${ }^{4}$. A consolidação de memórias traumáticas se enquadra como outro fator que altera a homeostase do SNC. Ter experiências traumáticas como, por exemplo: acidentes, morte de familiares, doenças crônicas, maus-tratos e abuso sexual, levam a formação de memórias de medo em estruturas cerebrais denominadas hipocampo e amígdala, regiões responsáveis pelo armazenamento e processamento de informações ${ }^{5}$. Essas lembranças impedem o bem-estar das pessoas quando em presença do estímulo que levou ao trauma, podendo desencadear quadros crônicos de distúrbios de ansiedade e depressão.

Atualmente o tratamento farmacológico para doenças psicológicas ocasiona altos índices de efeitos adversos e 
demoram muito para iniciarem efeitos benéficos ao quadro doentio. Naturalmente, novas alternativas terapêuticas para essas enfermidades são foco de estudos científicos e a neuroimunologia associada com a neuroimunomodulação começa a ser mais explorada6.

A alteração do funcionamento do sistema imunológico por estresse patológico pode levar a depressão, transtornos do humor e ansiedade, devido à intensa atividade de moléculas sinalizadoras do sistema imune ${ }^{7}$. Além disso, sabe-se que com o estímulo de processos inflamatórios in vivo, facilita déficits cognitivos, atrelados a perda substancial de memória ${ }^{8}$. Outra relação relevante se solidifica no aumento da concentração de bactérias da microbiota natural e o desenvolvimento de estresse, ansiedade e depressão ${ }^{9}$. Novos estudos centrados nesse assunto propõem a atualização de tratamentos para doenças psicológicas a partir de anti-inflamatórios, ou seja, tratamentos baseados no controle de mediadores inflamatórios ${ }^{9}$. Porém, mesmo que algumas alternativas nesse sentido estejam ganhando importância, o seu potencial terapêutico ainda não está totalmente esclarecido. Portanto, é consolidado que a neuroimunomodulação seja capaz de modular a homeostase do SNC e que esses sistemas possam trabalhar em conjunto no desenvolvimento de doenças psicológicas e neurodegenerativas.

Nesse sentido, este artigo de revisão objetiva identificar os principais mecanismos neuroimunológicos envolvidos na patogênese de processos psicológicos, tais como ansiedade 
e depressão, bem como, em doenças neurodegenerativas, como Mal de Alzheimer e Doença de Parkinson.

\section{MÉTODO}

Este artigo de revisão foi construído através de consultas em bancos de dados acadêmicos e dados epidemiológicos como SCIELO, SCIENCE DIRECT e PUBMED.

A consulta foi realizada utilizando como palavras-chave: "doenças neurodegenerativas", "doenças psicológicas", "sistema imunológico" "neuroimunomodulação" e "mecanismos cerebrais".

Artigos na língua inglesa e portuguesa, publicados nos últimos 15 anos, foram considerados para a preparação desta revisão.

\section{RESULTADOS E DISCUSSÃO}

Atualmente sabe-se que inúmeros estímulos provenientes do SNC são capazes de modular uma resposta imune e vice-versa. O eixo Hipotálamo-Pituitária Adrenal (HPA) é um dos responsáveis nessa troca de informação. Também participam desta relação alguns hormônios como: endorfinas, prostaglandinas, tireotrofinas, hormônio do crescimento e o Sistema Nervoso Autônomo Simpático $(\mathrm{SNAS})^{8}$. Além disso, a ativação do eixo HPA e a consequente produção dos hormônios glicocorticoides durante o processo de estresse são um dos principais mecanismos responsáveis 
pelas alterações da resposta imune corporal. Os hormônios glicocorticoides se mostraram capazes de inibir a transcrição de inúmeras citocinas, como de interleucina 1 (IL-1), IL-13, IL-5, IL-6, IL8, fator de necrose tumoral (TNF) e fator estimulante de colônia (GM-CSF). Além disso, estes hormônios se mostraram inibidores da migração/quimiotaxia de eosinófilos e de neutrófilos ${ }^{10}$. Foi observado que a presença de estresse foi diretamente relacionada com a leucocitose, diminuição no número de células NK, aumento na relação $\mathrm{CD} 4+/ C D 8+$ e diminuições nas atividades de células T e NK. Há nítida relação de processos inflamatórios periféricos com o aumento do estresse e alterações neurohumorais. O aumento de citocinas inflamatórias pode levar a ruptura da Barreira-Hemato Encefálica (BHE), gerando inflamação no SNC, e consequente disfunção psicológica e neuronal11.

O estresse está amplamente relacionado ao SNAS, com a ativação desse sistema ocorre a dispersão do hormônio noradrenalina nas extremidades nervosas simpáticas e à liberação de adrenalina trazida pela glândula adrenal. Os órgãos linfoides têm íntima relação com os filamentos simpáticos, a exemplo disso, pode-se citar: o baço, outros órgãos e células imunes, como o pulmão, macrófagos alveolares e linfócitos circulantes que detêm elevada densidade de $\beta$-adrenoceptores. Ensaios in vivo, a partir de camundongos expostos ao estresse, apresentaram uma maior liberação de $I L-1 \beta$ por macrófagos alveolares promovido pelo bloqueio antecipado da atividade do SNA e, 
em especial dos $\beta 2$-adrenoceptores, minutos antes da aplicabilidade do estresse ${ }^{8}$.

A psiconeuroimunologia fundamenta-se, preferencialmente, sobre a teoria de estresse. Os hormônios de estresse mais conhecidos como as catecolaminas (adrenalina e noradrenalina), os corticoides (cortisol, aldosterona) e muitos outros são, por si só, responsáveis por uma significativa parte da imunossupressão ${ }^{12}$. É de conhecimento que o estresse é dos principais vilões de alterações do humor como transtorno de estresse póstraumático, ansiedade e depressão.

Uma descoberta surpreendente da neurociência moderna que transformou profundamente essa nossa concepção dos sistemas nervoso e imunológico, é a de que células imunológicas produzem neurotransmissores e que neurônios, por sua vez, também secretam imunopeptídeos. Isso permite que ambos os sistemas possuam dispositivos para estabelecer uma comunicação e linguagem específica entre si, englobando uma via central, imune e endócrina ${ }^{13}$.

Em um estudo utilizando glicocorticoides em modelos animais de memórias traumáticas, percebeu-se que a administração do medicamento interferiu na consolidação dessas memórias, contribuindo para o enfraquecimento de fatos traumáticos nos animais ${ }^{14}$. Nesta mesma linha, o uso de anti-inflamatórios já se mostrou benéfico para doenças neurodegenerativas como Alzheimer e Parkinson, por exemplo. A diminuição do processo inflamatório retardou perdas neuronais pela diminuição da sedimentação proteica 
e diminuição do processo oxidativo, ambos característicos das patologias ${ }^{15}$. Nesse sentido, grupos científicos e clínicos acreditam que a diminuição de cascatas inflamatórias, devido ao uso de anti-inflamatórios, pode retardar o progresso de alterações neurológicas e humorais, entretanto os efeitos adversos relacionados ao uso destes medicamentos para esta finalidade divergem opiniões na literatura ${ }^{16}$.

Pacientes com transtorno depressivo maior apresentam alterações em marcadores imunológicos, como citocinas próinflamatórias e células inflamatórias, estando implicadas no estabelecimento das alterações humorais encefálicas ${ }^{17}$. Em modelos animais, indução e/ou administração de citocinas pró-inflamatórias produz um conjunto de mudanças de comportamento caracterizadas por diminuição do apetite, perda de peso, distúrbios do sono, atraso na atividade motora, menor interesse no ambiente físico e social e perda da libido, comuns observados em pessoas depressivas. Em voluntários humanos saudáveis, a indução de citocinas endógenas por endotoxinas está associada ao desenvolvimento transitório de humor deprimido, ansiedade e deficiências de memória. Em contraste, os sintomas neuropsiquiátricos, incluindo ansiedade, disforia, anedonia, fadiga, anorexia, desaceleração cognitiva e psicomotora geralmente ocorrem após um a três meses de terapia com citocinas. Esses sintomas característicos da depressão desaparecem pelo uso de medicamentos antidepressivos ${ }^{18}$.

A exposição a processos inflamatórios durante a 
gravidez pode induzir quadros de doenças psicóticas como esquizofrenia anos depois do nascimento da criança. De forma interessante, observou-se que a administração de interferon gama, uma citocina inflamatória, tem uma maior tendência a induzir quadros de depressão nesses pacientes. E ainda, doenças inflamatórias crônicas como, diabetes, obesidade, artrite, estão comumente relacionadas com 0 aparecimento de transtornos de ansiedade e depressão ${ }^{19}$.

Por mais que sejam tópicos de amplo debate na literatura, as evidências ainda divergem. Enquanto alguns estudos relatam que pessoas deprimidas mostram reduções das respostas imunes, aumentando a susceptibilidade a doenças infecciosas, outros demonstram o contrário.

Discute-se muito sobre a comunicação ativa e constante de bactérias com o SNC. O desenvolvimento da microbiota ocorre logo após o nascimento e está diretamente relacionado à fisiologia do hospedeiro, o desenvolvimento e morfogênese de órgãos e a manutenção do equilíbrio de tecidos $^{20}$.

Estudos mostram que a função cerebral pode ser diretamente interligada com a microbiota intestinal21,22. Um dos nervos cranianos, chamado de nervo vago, é de extrema importância para que a comunicação cérebro e microbiota aconteça, eixo fundamental que media diversos fatores comportamentais. Dessa forma, neurotransmissores sintetizados pela microbiota intestinal transpassam a mucosa do intestino e atuam no encéfalo ${ }^{21}$. São vários os neurotransmissores produzidos por espécies comensais 
como, por exemplo: Lactobacillus, Bifidobacterium - GABA; Escherichia, Bacillus, Saccharomyces - Noradrenalina; Candida, Streptococcus, Escherichia, Enterococcus Serotonina; Bacillus, Serratia - dopamina; LactobacillusAcetilcolina22. Porém, a alteração da microbiota intestinal pode ocasionar um processo de desequilíbrio, chamado de disbiose. Esse processo pode ser acarretado por variados fatores que venham modificar a composição da microbiota intestinal, favorecendo o crescimento e desenvolvimento de microrganismos patogênicos e contribuindo diretamente para a formação de estados patológicos e neurológicos, facilitando o início de transtornos psiquiátricos, ansiedade e depressão 21,22 .

Em contrapartida, a administração oral de probióticos microorganismos vivos não patogênicos - como: Saccharomycesboulardiie Saccharomycescerevisiae, fungos do tipo levedura, poderiam representar uma alternativa ao uso de medicamentos para estes distúrbios psiquiátricos ${ }^{21}$. Indo mais além, as bactérias intestinais são capazes de regular vários aspectos do sistema imune inato e adaptativo, sendo o trato gastrointestinal responsável por cerca de 60\% do sistema imunológico. As múltiplas interações entre o epitélio, os receptores e os micro-organismos intestinais estão constantemente remodelando o sistema imunológico local e sistêmico e influenciando diretamente o metabolismo do hospedeiro 20 .

Após décadas de pesquisas, percebeu-se uma forte associação entre infecções por microrganismos e o 
aparecimento de doenças neurodegenerativas. A doença de Alzheimer pode ser mais suscetível a partir de infecções bacterianas ${ }^{24}$. Diversos estudos demonstram, por exemplo, a presença de bactérias no cérebro de pacientes em idades avançadas, estando estes microrganismos correlacionados com as patologias e características da doença. A encefalite, também causada por esses microrganismos, produz danos ao sistema nervoso central, sendo associada à perda de memória e a redução dos processos cognitivos, de forma semelhante ao Alzheimer ${ }^{23}$. Além disso, a deposição de placas beta-amiloides e o acúmulo de proteínas tau ocorrem devido à infecção por microrganismos e observa-se um aumento de células gliaastrocitárias ao redor dos emaranhados proteicos característicos da doença ${ }^{24}$. Mostrouse que o aumento da resposta inflamatória central pode levar a formação de espécies reativas de oxigênio e a depleção de neurônios dopaminérgicos e colinérgicos, influenciando na patologia da doença de Parkinson e Alzheimer.

Há produção da citocina pró-inflamatória chamada interleucina-1 beta (IL-1B), envolvido na defesa do organismo contra a infecção, sendo esta utilizada como um alvo clínico para a artrite reumatoide ${ }^{24}$. Algumas drogas que interrompem a produção de IL-1B, tais como os medicamentos contra a artrite reumatoide, podem também revelar-se benéficas para pacientes com o mal de Alzheimer. Devido a isso, é possível que as drogas que bloqueiam cascatas inflamatórias, possam fornecer algum benefício às doenças neurodegenerativas ${ }^{24}$. 
Em modelos in vivo de estresse, observou-se que o estresse repetido pareceu causar uma reação imune nos cérebros de roedores, levando à inflamação. Entre 10-28 dias após a exposição ao estresse, o processo de neurogênese no hipocampo significativamente declinou, induzindo déficits cognitivos e perda de memória de curta duração. Os cientistas administraram compostos antiinflamatórios nos roedores, e eles foram capazes de controlar a perda de memória observada 25 .

Torna-se claro que o sistema nervoso e o sistema imune trabalham em conjunto, tanto na regulação de vias, quanto no desenvolvimento de patologias. Alterações do funcionamento do sistema imunológico na presença de uma doença podem desencadear depressão, transtornos do humor e ansiedade, devido à intensa atividade de moléculas sinalizadoras imunológicas. Os novos estudos centrados nesse assunto propõem a atualização de tratamentos para depressão, ansiedade, transtorno pós-traumático a partir da modulação inflamatória. Porém, mesmo que algumas alternativas estejam ganhando importância, ainda não se tem exatamente claro seu perfil terapêutico relacionado a essas patologias. Sugere-se aqui que, possivelmente, a neuroimunomodulação possa garantir uma maior estabilidade ao SNC, sendo uma importante abordagem terapêutica para doenças psicológicas e neurodegenerativas. 


\section{CONCLUSÃO}

Os trabalhos na área da neuroimunomodulação têm contribuído de forma marcante para o entendimento da regulação do sistema imune sobre o desencadeamento de alterações neurológicas. Com essa conversa entre os dois sistemas, se estabelece a resposta para diversas sinalizações celulares e moleculares que podem gerar quadros patológicos. Sabe-se que o uso de anti-inflamatório foi capaz de modular a patogênese de doenças mentais, como a depressão. $\mathrm{O}$ correto esclarecimento sobre vias $e$ mediadores envolvidos neste processo, possibilitará abordagens terapêuticas mais eficazes para doenças psicológicas e neurodegenerativas.

\section{AGRADECIMENTOS}

Ao Centro Universitário UniFBV/Wyden pelo suporte estrutural e científico para a realização deste artigo. Ao corpo de docentes do Centro Universitário UniFBV pelo conhecimento compartilhado.

\section{REFERÊNCIAS}

1. Louveau A, Smirnov I, Timothy JK, Eccles JD, Rouhani SJ, Peske JD, et al. Structural and functional features of central nervous system lymphatic vessels. Nature 2015; $523: 337-41$.

http://doi.org/10.1038/nature14432

2. Marques AH, Cizza G, Sternberg E. Interações imunocerebrais e implicações nos transtornos psiquiátricos. Braz J Psychiatr 2007;29:s27-32. http://doi.org/10.1590/S1516-44462007000500006 3.Falco AD, Cukierman DS, Hauser-Davis RA, Rey NA. Alzheimer's disease: etiological hypotheses and treatment perspectives. Quím 
Nova 2016;39:63-80. http://doi.org/10.5935/0100-4042.20150152

4.Tiwari PC, Pal R. The potential role of neuroinflammation and transcription factors in Parkinson disease. Dial Clin Neurosci 2017;19:71. http://doi.org/10.31887/DCNS.2017.19.1/rpal

5. Oliveira HM, Albuquerque PBD. Explanatory Mechanisms of False Memories in DRM Paradigm. Psicol Refl Crit 2015;28:554-64. http://doi.org/10.1590/1678-7153.201528314

6. Bauer M, Severus E, Möller HJ, Young AH. Pharmacological treatment of unipolar depressive disorders: summary of WFSBP guidelines. Int J Psychiatr Clin Pract 2017;21:166-76. http://doi.org/10.1080/13651501.2017.1306082

7.Sink KM, Holden KF, YAFFE K. Pharmacological treatment of neuropsychiatric symptoms of dementia: a review of the evidence. Jama 2005;293:596-608. http://doi.org/10.1001/jama.293.5.596

8.Alves GJ, Palermo-Neto J. Neuroimunomodulação: sobre o diálogo entre os sistemas nervoso e imune. Braz J Psychiatr 2007;29:4. http://doi.org/10.1590/S1516-44462006005000052

9. Medeiros AC, Maynard DDC. A influência intestinal no desenvolvimento de processos depressivos e o uso de probióticos como tratamento. Brasília (Monografia). Brasília: Centro Universitário de Brasília; 2019.

https://repositorio.uniceub.br/jspui/bitstream/prefix/13492/1/216057 73.pdf

10.Vandewalle J, Luypaert A, Bosscher KD, Libert C. Therapeutic mechanisms of glucocorticoids. Trends Endocrinol Metab 2018;29:4254. http://doi.org/10.1016/j.tem.2017.10.010

11.Machado APR, Carvalho IO, Rocha Sobrinho HM. Neuroinflamação na doença de Alzheimer. Rev Bras Mil Cienc 2020;6:30-8. http://doi.org/10.36414/rbmc.v6i14.33

12. Calcia MA, Bonsall D, Bloomfield P, Selvaraj S, Barichello Howes, OD. Stress and neuroinflammation: a systematic review of the effects of stress on microglia and the implications for mental illness. Psychopharmacol 2016;233:1637-50. http://doi.org/10.1007/s00213016-4218-9

13.Pinho-Ribeiro FA, Jr Verri WA, Chiu IM. Nociceptores sensoriais neurônio-interação imunológica na dor e inflamação. Trends Immunol 2017;38:5-19. http://doi.org/10.1016/j.it.2016.10.001

14.Souza CACD, Sander PDT. Déficits variados após lesões neurológicas bacterianas ou virais: implicações clínicas, periciais e psicossociais. Psychi Onl Bras 2003;8. http://www.polbr.med.br/ano03/artigo1103_b.php

15.Zhang QS, Heng Y, Yuan $\mathrm{YH}$, Chen $\mathrm{NH}$. Pathological a-synuclein exacerbates the progression of Parkinson's disease through microglial activation. Toxicol Lett 2017;265:30-7. http://doi.org/10.1016/j.toxlet.2016.11.002

16.Quevedo J, Feier G, Agostinho FR, Martins MR, Roesler R. Memory consolidation and post traumatic stress disorder. Rev Bras Psiquiatr 2003;25:25-30. http://doi.org/10.1590/S1516-44462003000500007 17. Hodes GE, Kana V, Menard C, Merad M, Russo SJ. Neuroimmune 
mechanisms of depression. Nat Neurosci 2015;18:1386-93. http://doi.org/10.1038/nn.4113

18.Gold SM, Irwin MR. Depression and immunity: inflammation and depressive symptoms in multiple sclerosis. Immunol Allergy Clin North Am 2009;29:309-20. http://doi.org/10.1016/j.iac.2009.02.008 19.Christian L. Effects of stress and depression on inflammatory immune parameters in pregnancy. Am J Obstet Gynecol 2014;211:275-7. http://doi.org/10.1016/j.ajog.2014.06.042

20.Gonçalves M. Microbiota: Implicações na imunidade e no metabolismo (Dissertação). Lisboa: Universidade Fernando Pessoa, 2014. https://bdigital.ufp.pt/bitstream/10284/4516/1/PPG 21951.pdf 21. Nesi GA, Franco MR, Capel LMM. A disbiose da microbiota intestinal, sua associação no desenvolvimento de doenças neurodegenerativas e seus possíveis tratamentos. Brazil J Develop 2020;6:63306-26. http://doi.org/10.34117/bjdv6n8-677

22.Silvestre C. O diálogo entre o cérebro e o intestino - Qual o papel dos probióticos? (Dissertação) Lisboa: Faculdade de Medicina da Universidade de Lisboa, 2015. https://repositorio.ul.pt/bitstream/10451/26287/1/CarinaRFSilvestre. pdf

23.Itzhaki RF, Lathe $R$, Balin BJ, Ball M, Portador E, Braak $H$, et al. Microbes and Alzheimer's disease. J Alzheimers Dis 2016;51:979. http://doi.org/10.3233/JAD-160152

24.Barreiros A, David J. Estresse oxidativo: relação entre gerações de espécies reativas e defesa do organismo. Quím Nova 2006;29:113-23. http://doi.org/10.1590/S0100-40422006000100021

25.Radulovic J, Jovasevic V, Meyer MAA. Neurobiological mechanisms of state-dependent learning. Curr Opin Neurobiol 2017;45:92-8. http://doi.org/10.1016/j.conb.2017.05.013 